\title{
Refocus the Recombinant DNA Advisory Committee
}

\author{
Xandra O Breakefield
}

\begin{abstract}
The Recombinant DNA Advisory Committee, formed in 1974, was originally charged with advising on potential risks of recombinant technology and took on review of clinical gene therapy protocols in the late 1980s. But as gene therapy has made headway with safety, it's time to shift the committee's mission toward broader ethical and technical issues linked to the field.
\end{abstract}

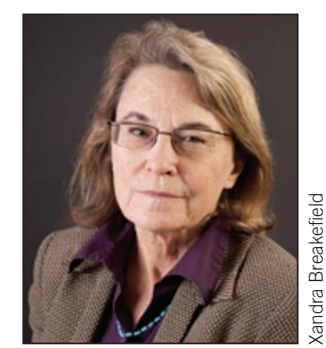

As in the development of any new class of therapeutics, there were many challenges and concerns when the field of gene therapy emerged. To this end, the director of the US National Institutes of Health (NIH) formed the Recombinant DNA Advisory Committee (RAC) in 1974 to provide a forum for public discussion of emerging methodologies within this field and to advise the NIH director on issues concerning risks to public safety. And, since its inception in 1996, the American Society of Gene \& Cell Therapy (ASGCT) and its members have worked with the RAC to improve the efficacy and reduce any risks associated with gene transfer in clinical protocols.

Over time, the gene and cell therapy community has evolved in technical sophistication with increasing numbers of trials (over 1,000 clinical trials internationally), and it has reached the stage of commercialized products for several diseases. In recent years, investigators have reported clear benefit in clinical trials using gene and cell therapies for a number of diseases, including hereditary blindness, immune deficiencies and Parkinson's disease. But the RAC continues to use the same formula for review of individual, committee-selected clinical protocols. All proposed clinical trials that used NIH funding in their development must be submitted in full, and the RAC selects about $20 \%$ a year for detailed on-site review.

In the opinion of many highly regarded experts who are members of ASGCT, the current modus operandi of the RAC is not optimal. In the years since the first gene therapy trials were performed, the US Food and Drug Administration (FDA), the Office for Human Research Protections, institutional review boards and institutional biosafety committees have become very experienced and knowledgeable and are fully competent to carry out critical regulatory review of study protocols within the gene and cell therapy field. In addition, reviews of past performance and consideration of safeguards have been put in place since RAC reviews began, such as data safety and monitoring boards for early phase trials, with adverse events reported immediately to the FDA. The current mandate of the RAC adds another layer of nonregulatory review to protocols, thereby increasing time and expense. As it stands, the RAC clinical review process is basically duplicative of that of regulatory agencies, with the difference being that it is carried out in an open forum with representatives from the public. The RAC also comments on the sufficiency of preclinical data and requests new preclinical data to be generated in some cases. As there is no real coordination between the RAC and the FDA on this, investigators are sometimes in 'double jeopardy' trying to serve two masters with differing opinions.

Given these circumstances, in March 2012, the ASGCT board of directors, with the advice of an internal panel of experts, voted to recommend that this system be streamlined. Specifically, the president at the time, Jude Samulski, director of the Gene Therapy Center at the University of North Carolina-Chapel Hill, and I, as president-elect, sent a letter to the NIH in which we recommended that "the RAC would terminate review of individual clinical protocols and would instead identify new areas of research that require a public forum for discussion and review." This position reflects our mission-similar to that of the new NIH National Center for Advancing Translational Sciences-to facilitate the development of effective treatments and bring them to patients as efficiently as possible.

Rather than have the RAC duplicate the efforts of regulatory agencies, a more effective use of resources would be to use its convening power and collective wisdom to facilitate workshops for new investigators entering the field and to discuss common operating procedures, as well as ethical and regulatory issues. The RAC should continueas it has done so well in the past-to organize focused, in-depth symposia with experts and lay people on newly arising and problematic issues in the field. Such issues would include new vector designs and their associated biosafety risks; the societal aspects of expanded treatment indications such as prenatal therapy; the use of gene delivery for athletic enhancement; and the evaluation of safety issues.

In 2010, for example, together with the European Network for the Advancement of Clinical Gene Transfer and Therapy (CliniGene), the RAC held a public symposium in Bethesda, Maryland, to discuss oncogenic events due to genome-integrating vectors and how to reduce them. In addition, public discussion is urgently needed on the use of a common vector for multiple indications, for instance, ocular gene transfer across diseases of different etiologies when there are only a few patients of each specific subtype and funding and resources are not available for repeated in-depth toxicology studies for each subtype. To ensure that 'no patient is left behind' and 'no disease is too small', the field needs a clear consensus on how best to bring successful approaches to all patients who stand to benefit from these therapies.

Early public concerns about potential negative societal outcomes of gene therapy - such as contamination of the germ line or generation of new pathologic agents-have been allayed by the absence of such events in nearly two decades of clinical trials. In fact, the ASGCT perceives that the public is becoming more concerned that large public investment in biomedical research has been slow to lead to improved treatments for illness. We would therefore welcome a decision by NIH director Francis Collins to initiate an external review of the RAC to modify its operating procedures and bring it into alignment with the current era of rapid advances in gene and cell therapy.

Xandra O. Breakefield is president of the American Society of Gene \& Cell Therapy and professor of neurology at the Harvard Medical School in Boston, Massachusetts, USA. 